\title{
Un enfoque comparativo sobre la integración y apertura comercial en el crecimiento económico de la Unión Europea y América Latina Alberto Villagra-Piña
}

Universidad Nacional Autónoma de México, Posgrado de la Facultad de Economía Miguel Ángel Mendoza-González*

Universidad Nacional Autónoma de México, Posgrado de la Facultad de Economía Luis Quintana-Romero

Universidad Nacional Autónoma de México, FES Acatlán

(Recibido 17 de enero de 2017, aceptado 25 de julio de 2017)

\begin{abstract}
Resumen
El objetivo de esta investigación consiste en probar si la integración económica y la apertura comercial en los países de la Unión Europea (UE) y América Latina (AL), ayudaron a aliviar los desequilibrios de balanza de pagos previos. La principal contribución del trabajo descansa en el análisis comparativo entre la UE y AL en dos periodos distintos de tiempo (de acuerdo con su integración económica) utilizando la técnica de panel cointegrados de Westerlund (2007 y 2008) para probar la validez de la ley de Thirlwall. Con los resultados, se encontró que los precios relativos no influyeron de manera relevante en los periodos de integración y apertura comercial, por lo que resultó más adecuado trabajar con el multiplicador dinámico del comercio de Harrod. Es importante mencionar que, aunque el enfoque contrafactual se aplica a momentos históricos de Europa y América Latina distintos, con sus resultados se pudo concluir que la integración económica ayudó a aliviar los desequilibrios de balanza de pagos en los países europeos y la apertura comercial provocó un mayor crecimiento compatible con el equilibrio de balanza de pagos, para la mayoría de los países de América Latina.
\end{abstract}

Clasificación JEL: F15, F13, F17, F43.

Palabras clave: Integración económica, Apertura comercial, Crecimiento económico, Restricción de Balanza de Pagos, Países de Unión Europea y América Latina.

\section{A Comparative Approach to Integration and Trade Openness in the Economic Growth of the European Union and Latin America}

\begin{abstract}
The aim of this research is to prove whether Economic Integration and Trade Openness in countries of the European Union (EU) and Latin America (LA) helped alleviate previous Balance of Payments imbalances. The main contribution of the work consists on the comparative analysis between the EU and LA in two different periods of time (according to their economic integration) using the Westerlund panel cointegration technique (2007 and 2008) to prove the validity of Thirlwalls Law. The results show that the relative prices are not

* División de Posgrado de la Facultad de Economía de la Universidad Nacional autónoma de México, Ciudad Universitaria, Edificio de Posgrado, cubículo 213, segundo piso, C.P. 10450, Delegación Coyoacán, Ciudad de México, México, Teléfono: + 52 (55) 56-22-18-88 ext. 4889. E-mail: mendozag@unam.mx
\end{abstract}


relevant in periods of Economic Integration and Trade Openness, consequently it was more appropriate to work with Harrods Dynamic Trade Multiplier. It is important to mention that, although the counterfactual approach is applied to different historical moments in Europe and Latin America, the results showed that the Economic Integration helped alleviate the Balance of Payments imbalances in European countries and Trade Openness caused a greater growth compatible with the Balance of Payments equilibrium for most Latin American countries.

JEL Classification: F15, F13, F17, F43.

Key Words: Economic Integration, Trade Openness, Economic Growth, Balance-of-Payments Restraint, European Union Countries and Latin America.

\section{Introducción.}

Los procesos de integración económica o apertura comercial en la Unión Europea (UE) y América Latina (AL) se dieron con el objetivo principal de eliminar obstáculos a los flujos comerciales, en la búsqueda de aumentar las exportaciones netas y con ello afectar positivamente el crecimiento económico en los países que integran cada zona (Gazol, 2008; Tamames, 2006). Sin embargo, los posibles efectos positivos estuvieron condicionados por las estructuras de los flujos comerciales antes y después de la entrada en vigor de los acuerdos en forma del libre comercio (Calvo, 2003; Funck \& Pizzati, 2003; Muñiz, 2005; Ocegueda, 2007; Pastor, 1993; Schiff \& Winters, 2003). El planteamiento de Thirlwall (Thirlwall, 2003; Thirlwall, 2003; Thirlwall, 1997; Thirlwall, 1979) como el de Harrod (Bairam, 1988; Bairam, 1993; McCombie, 1997; Santos \& Thirlwall, 1999), son importantes para probar que bajo ciertas condiciones estructurales de balanza de pagos, el proceso de integración económica genera mayor crecimiento económico. Pero si tales condiciones no se cumplen entonces el escenario de restricciones de balanza de pagos condiciona el crecimiento económico por debajo del potencial (Alonso \& Garcimartín, 1999; Ashok, 2006; Atesoglu, 1994; Bajo-Rubio \& Díaz, 2009; Gairuzazmi, 2006; Landesmann \& Pöschl, 1996; Thirlwall, 1979). El objetivo principal de esta investigación consiste en determinar la importancia de la apertura comercial e integración económica en el crecimiento económico de largo plazo de cada región. Nuestro análisis se enfoca en probar que las restricciones por Balanza de Pagos se agudizó en AL a pesar de los múltiples procesos de integración en la zona, mientras que en UE se aliviaron tales restricciones debido al proceso añejo y ordenado de integración económica europeo. En particular, el enfoque comparativo que utilizamos, permite analizar como los acuerdos entre países en procesos de integración económica de la unión europea provocaron crecimientos económicos por arriba del potencial en la mayoría de ellos, debido principalmente a las menores restricciones de balanza de pagos y agudizaron las restricciones previas de balanza de pagos en la mayoría de los países en América Latina, que condicionaron las perspectivas de crecimiento económico por debajo de su potencial.

Para diseñar el enfoque comparativo entre los procesos de integración y apertura comercial en ambas zonas, se consideró tanto las características de los países en los bloques de uniones económicas como en la delimitación temporal de los procesos de integración. Aunque los grados de integración que experimentaron ambas zonas son totalmente distintos en tiempo y espacio, se propone utilizar un enfoque histórico para identificar la similitud en grado y 
etapa de integración económica en la Unión Europea y en América Latina. Para ello, se tomó como referencia histórica el proceso de preferencias arancelarias en Europa alrededor de 1957, fecha en que entró en vigor la Comunidad Económica Europea (CEE), su consolidación hacia 1968 y su completitud en 1992 con la conformación de lo que hoy conocemos como "Unión Europea" (UE). Mientras que para América Latina, se consideró la transformación de la Asociación Latinoamericana de Libre Comercio (ALALC) en Asociación Latinoamericana de Integración (ALADI) en 1980 y su ruptura en el Grupo de los Tres (G 3. México, Colombia y Venezuela) en 1994, el Mercado Común del Sur (Mercosur) en 1991 y la consolidación de la propuesta de la creación del Área de Libre Comercio de Sudamérica (ALCSA) en 1994. Además por supuesto, de la entrada en vigor del Tratado de Libre Comercio de América del Norte (TLCAN) en 1994.

Con el marco histórico anterior la metodología econométrica empleada considera un periodo previo y otro posterior a los procesos de integración económica y/o apertura comercial para analizar tanto las condiciones como las consecuencias en el crecimiento económico en los países de la Unión Europea y América Latina. Para la Unión Europea se definió como periodos previo y posterior a la integración económica, de 1957 a 1968 (once años) y de 1969 a 1992 (veintitrés años), mientras que los periodos para América Latina fueron de 1980 a 1994 (catorce años) y de 1995 a 2012 (diecisiete años), respectivamente en cada caso. Una vez definidos los periodos de comparación de los procesos de integración económica, se aplicaron modelos de crecimiento económico con restricciones de balanza de pagos para analizar las condiciones estructurales de los flujos comerciales previos y posteriores a la apertura comercial, y su impacto en el crecimiento económico potencial en la Unión Europea y América Latina.

Esta investigación se integra con esta introducción, en el segundo apartado se presentan las evidencias empíricas tanto para los países de la Unión Europea como de América Latina. En el apartado tres, se presenta la metodología que incluye la especificación del modelo de Thirlwall y Harrod, la estimación de cointegración en panel de Westerlund (2007 y 2008), con efectos aleatorios y dependencia de sección cruzada entre los países, para las exportaciones e importaciones de las dos regiones. En el aparatado cuatro se exponen los resultados. Y finalmente se presentan las conclusiones finales.

\section{Evidencia empírica para la Unión Europea y América Latina.}

El planteamiento central del modelo de crecimiento restringido por la balanza de pagos, conocido también como ley de Thirlwall, consiste en que ningún país puede crecer más rápido de lo que le permite el equilibrio en su balanza de pagos en su cuenta corriente a menos que tenga capacidad suficiente de financiar grandes y crecientes déficits, los cuales tienen un límite en relación con el ingreso. Es así, que desde el planteamiento inicial de Thirlwall (1979), se ha tratado de verificar si se cumple esta restricción por balanza de pagos. En esta sección revisaremos algunos estudios que analizan la Ley de Thirlwall tanto en Europa como América Latina y principalmente aquellos que incorporan de alguna manera la apertura comercial para determinar el crecimiento.

\subsection{Evidencia empírica para Unión Europea}

Thirlwall en 1979 como parte de su planteamiento inicial analizó los resultados de su modelo considerando el crecimiento del producto y de las exportaciones 
para los periodos de 19561976 y 19511973 para diversos países, en su mayoría europeos utilizando la elasticidad ingreso de la demanda por importaciones de Houthakker y Magee (1969) y aplicando la fórmula del multiplicador dinámico del comercio de Harrod. Los resultados que obtuvo muestran una tendencia general de los países europeos, que mantuvieron un crecimiento económico consistente con el equilibrio de la balanza de pagos; esto es, una tasa de equilibrio más alta que la tasa observada. Este resultado indica la existencia de superávits comerciales en todos los países, como lo es el caso japonés, que creció más rápido que otros países.

Bairam (1988) realizó un estudio similar para diecinueve países de Europa y América del Norte utilizando datos de la "Economic Survey of Europe" de 1970 1986 a precios de 1975 y el ingreso mundial representado por la media ponderada de las tasas de crecimiento del ingreso doméstico de los diecinueve países. Los resultados que obtuvo de las elasticidades ingreso de las exportaciones fueron con el signo correcto y estadísticamente diferente de cero, excepto Alemania, Irlanda y Turquía. En cuanto a las elasticidades precio de las exportaciones, la mayoría fueron con el signo correcto, pero pequeñas y no fueron significativas lo que quiere decir que el efecto de los precios no fue importante. En lo referente a las elasticidades ingreso de las importaciones $(\pi)$ tienen el signo correcto, son estadísticamente significativas y más grandes que la unidad, mientras que las elasticidades precio, no fueron significativas o tienen el signo incorrecto, ello también implica que las elasticidades precio son pequeñas en comparación con las elasticidades ingreso. Los resultados obtenidos por Bairam comprueban la Ley de Thirlwall y sugieren que todo el crecimiento económico de los países está determinado por el multiplicador dinámico del comercio de Harrod, ello porque depende principalmente de los valores de las elasticidades ingreso.

\subsection{Evidencia empírica para América Latina}

Moreno Brid y Esteban Pérez (1999) realizaron una prueba del modelo restringido por la balanza de pagos mediante cointegración de Johansen para cinco economías de América Central (Costa Rica, Guatemala, El Salvador, Honduras y Nicaragua). En todos los casos, las elasticidades ingreso estimadas de las importaciones fueron positivas entre valores desde 1.10 (Costa Rica) hasta 3.70 (Honduras) y como se esperaba, las elasticidades precio de las importaciones fueron siempre negativas en un rango de -0.44 (El Salvador) hasta -1.63 (Nicaragua). Los parámetros estimados de los vectores de cointegración también sugieren que las exportaciones son la variable explicativa más relevante de la tasa de crecimiento del producto. Además, los resultados indican que los países con las tasas de crecimiento de largo plazo del PIB más rápidas compatibles con el equilibrio de balanza de pagos en el periodo 19501996 mantuvieron elasticidades ingreso de las importaciones bajas y un elevado crecimiento de las exportaciones.

Thirlwall y Pacheco López (2006) realizaron un estudio para un conjunto de 17 países de América Latina para el periodo de 1977 a 2002. Estimaron un modelo de panel para obtener la elasticidad ingreso de las importaciones de toda la región y poder comparar la tasa de crecimiento real observada y la que predice el modelo para todo el periodo. Los resultados indican que la elasticidad ingreso de las importaciones es igual a 2.29 y la elasticidad precio de las importaciones de -0.069 , ambas tienen el signo esperado; la tasa 
de crecimiento promedio de las exportaciones $(x)$ para todos los países y todos los años es de 5.49 por ciento anual. La tasa de crecimiento compatible con el equilibrio en la balanza de pagos está determinada entonces por el multiplicador dinámico del comercio de Harrod, $\left(y_{b}=x / p\right)$, que para el conjunto de América Latina fue de 2.4 por ciento; mientras, que la tasa de crecimiento observada fue de 2.67 por ciento. Como conclusiones, sostienen que el crecimiento de muchos de los países de América Latina fue cercano a su tasa de crecimiento compatible con el equilibrio en sus balanzas de pagos, que no han cambiado significativamente pues permanece en alrededor de 2.5 por ciento anual. La experiencia individual es mixta, para algunos países se muestra un marcado incremento, en otras permanece constante y el resto es errático. La liberalización comercial sólo elevaría sustancialmente el crecimiento del producto si también logra un aumento en el crecimiento de las exportaciones más que proporcionalmente al registrado por la elasticidad ingreso de las importaciones; hechos que no ocurrieron en la mayoría de los países de América Latina. Por todo lo anterior, la liberalización comercial no ha sido exitosa en América Latina, en parte por una rápida liberalización que no dio tiempo a las economías internas a un ajuste de las exportaciones y la imposibilidad de poder competir con las importaciones, para ser exitosa necesita implementarse con una estrategia previa de comercio.

En el caso del estudio de Ferreira y Canuto (2003) se estimó un modelo de rezago distribuido para la economía brasileña de 1949 a 1999 encontrando evidencia fuerte sobre la Ley de Thirlwall, al obtener una elasticidad ingreso de las importaciones positiva y mayor que 1 y tasas de crecimiento observadas e hipotéticas muy cercanas reforzando el modelo. Lanzafame (2014) estima un modelo de panel con rezago distribuido para 22 países de la OCDE, incluyendo varios de la UE y a México. Con datos anuales de 1960 a 2010 y comprueba que la ley de Thirlwall es válida indicando además que el crecimiento debe ser consistente con el equilibrio de la balanza de pagos y que en el largo plazo está determinada por la demanda.

En el caso particular de México se ha mostrado que la apertura comercial también contribuyó a la creación de déficits en cuenta corriente, que han incrementado la fragilidad financiera, cambiaria y fiscal (Martínez, et.al. 2015). Morones (2016) estima un modelo de cointegración con corrección de error con choques estructurales endógenos para la economía mexicana de 1993 a 2014 y encuentra que el crecimiento de la economía mexicana se encuentra restringido por la balanza de pagos con una elasticidad ingreso de las importaciones de 2.27 y 2.24 para la elasticidad ingreso de las exportaciones soportando la validez de la ley de Thirlwall.

\section{Metodología}

\subsection{Modelo de Crecimiento económico restringido por la balanza de pagos}

El modelo de Thirlwall, sin flujos de capital, que utilizamos se especifica para un grupo de países con condiciones iniciales diferentes, que se comportan y reaccionan de forma heterogénea en los mercados internacionales. $Y$, donde sus rutas de crecimiento se determinan por el cumplimiento de las condiciones de equilibrio en cuenta corriente, donde la suma del crecimiento de los precios en moneda nacional $\left(p_{d, i t}\right)$ y cantidades de las exportaciones $\left(x_{i t}\right)$ es igual a 
la suma del crecimiento de los precios de bienes de competidores en moneda extranjera $\left(p_{f, i t}\right)$ y cantidades de las importaciones $\left(m_{i t}\right)$ más la depreciación del tipo de cambio medido como el precio en moneda nacional de la moneda extranjera $\left(e_{i t}\right)$, como se especifica en la ecuación 1:

$$
p_{d, i t}+x_{i t}=p_{f, i t}+m_{i t}+e_{i t}
$$

Donde los $i^{\prime} s$ países van de $1 \cdots n$ en los $t$ momentos en el tiempo de $1 \cdots T$. En un planteamiento general, los factores de demanda de las exportaciones son los determinantes principales en la explicación del crecimiento de largo plazo. En esta especificación, las exportaciones para cada uno de los $i^{\prime} s$ países $\left(x_{i t}\right)$ son una función positiva del incremento de los precios relativos medidos en una moneda común $\left(p_{(f, i t)}+e_{i t}-p_{(d, i t)}\right)$ y del ingreso de un economía líder $\left(y_{l t^{*}}\right)$. Donde los precios relativos se incrementan por el aumento de los precios de los bienes de los competidores medidos en moneda común $\left(p_{f, i t}\right)$ y de la depreciación del tipo de cambio $e_{i t}$, y se reducen por el crecimiento de los precios internos $\left(p_{d, i t}\right)$. El crecimiento promedio de las exportaciones heterogéneas de cada país, que no dependen del crecimiento de los precios relativos y del ingreso de la economía líder, se mide por la constante $\beta_{(0, i)}$ y la reacción heterogénea a los mercados internacionales por cambios en los precios relativos y del ingreso externo se miden con elasticidades individuales $\eta_{i}$ y $\varepsilon_{i}$ respectivamente; ver ecuación 2.

$$
x_{i t}=\beta_{0, i}+\eta_{i}\left(p_{f, i t}+e_{i t}-p_{d, i t}\right)+\varepsilon_{i}\left(y_{l t}^{*}\right)
$$

En tanto la demanda de importaciones $m_{i t}$ es una función negativa de los precios relativos $p_{f, i t}+e_{i t}-p_{d, i t}$ y positiva del ingreso nacional $\left(y_{(i, t)}\right)$. En este caso se espera que el crecimiento de las importaciones se determine por una caída de los precios de los bienes de los competidores $\left(p_{f, i t}\right)$ y por la apreciación del tipo de cambio $\left(e_{i t}\right)$, por un incremento de los precios internos $p_{d, i t} \mathrm{y}$ del ingreso nacional $\left(y_{i, t}\right)$. De la misma manera que en las exportaciones, las importaciones dependen de un crecimiento promedio estructural heterogéneo de cada país independiente de los precios relativos y del ingreso nacional, que se mide por la constante $\alpha_{0, i}$ y la reacción heterogénea a los cambios en los precios relativos y del ingreso nacional se miden con elasticidades individuales $\psi_{i} \mathrm{y} \pi_{i}$ respectivamente; ver ecuación 3 .

$$
m_{i t}=\alpha_{0, i}-\psi_{i}\left(p_{f, i t}+e_{i t}-p_{d, i t}\right)+\pi_{i}\left(y_{i, t}\right)
$$

$\mathrm{Al}$ sustituir las ecuaciones de las exportaciones e importaciones en la condición de equilibrio y despejando con respecto al crecimiento nacional, se obtiene la tasa de crecimiento del ingreso nacional consistente con el equilibrio de la balanza de pagos $\left(y_{B}\right)$; ver ecuación 4 :

$$
y_{B, i t}=\left[\left(\beta_{0, i}-\alpha_{0, i}\right)+\left(1-\left(\eta_{i}+\psi_{i}\right)\right)\left(p_{d, i t}-p_{f, i t}-e_{i t}\right)+\varepsilon\left(y_{l t}^{*}\right)\right] / \pi_{i}
$$

De la ecuación 4 se obtienen las siguientes inferencias: 1) Los países pueden mantener un crecimiento económico consistente con el equilibrio de balanza de pagos, dependiendo del crecimiento independiente de exportaciones 
e importaciones. Si el crecimiento de las exportaciones netas independientes son cero o estadísticamente igual a cero $\beta_{0, i}-\alpha_{0, i}=0$, entonces la ruta de crecimiento económico dependen de los términos de intercambio reales, del ingreso de la economía líder y de las elasticidades individuales medidas por las funciones de exportaciones e importaciones ${ }^{1}$. Si el crecimiento de las exportaciones netas es positivo $\beta_{0, i}-\alpha_{0, i}>0$, permite que la ruta de crecimiento económico con equilibrio de balanza de pagos sea factible y que sea mayor por los términos de intercambio y mejores condiciones de la economía líder internacional. $Y$, por último las posibilidades de crecimiento económico sostenido y consistente con balanza de pagos se reducen, cuando el crecimiento de las exportaciones netas independientes son negativas $\left.\beta_{0, i}-\alpha_{0, i}<0 ; 2\right)$ En el caso de una mejoría de los términos de intercambio reales $\left(p_{d}-p_{f}-e\right)>0$, la tasa de crecimiento económico será consistente con el equilibrio de la balanza de pagos; Thirlwall (2003) le llamó el "efecto términos de intercambio puro sobre el crecimiento del ingreso real"; 3) Si la suma de las elasticidades precio es mayor que la unidad $[(\eta+\psi)>1]$, entonces la tasa de crecimiento económico esperada es menor al de equilibrio de la balanza de pagos. En tal esquema, de suma de elasticidades precio mayor a la unidad y precios constantes, solamente aumentará la tasa de crecimiento de equilibrio de la balanza de pagos por una depreciación, que es equivalente a la condición MarshallLerner; 4) la tasa de crecimiento de equilibrio de la balanza de pagos tiene una relación inversa con la elasticidad ingreso de demanda por importaciones $\pi$; y, 5) En el supuesto de que los precios relativos permanezcan constantes la ecuación de equilibrio se reduce en $\left.y_{B}=\left[\varepsilon\left(y^{*}\right)\right] / \pi=x / \pi\right]$, que es la versión dinámica del multiplicador del comercio de Harrod (Bairam, 1988; Bairam, 1993; McCombie, 1997; Santos \& Thirlwall, 1999), donde el crecimiento económico es igual a la tasa de crecimiento de las exportaciones entre la propensión marginal a importar (Thirlwall, 2003; Thirlwall, 1997).

\subsection{Datos}

En la estimación del modelo de Thirlwall y Harrod se utilizaron las variables referentes al PIB, Importaciones $(M)$ y Exportaciones $(X)$ a precios constantes para los países de AL y la UE. Para la construcción de las variables se utilizaron los indicadores por país con información del Penn World Table (PWT) la versión 8.1 (Feenstra, Inklaar, \& Timmer, 2015). Para calcular el crecimiento económico se utilizó el PIB a precios de paridad de compra reales de 2005, que en el PWT se identifica como rgdpna; para las exportaciones e importaciones se utilizaron las variables que están en proporciones $\left(\operatorname{csh}_{x} \mathrm{y}\right.$ $c s h_{m}$ ) y se multiplicaron por el PIB; el tipo de cambio con respecto al dólar es igual a $x r$; los precios se miden con los deflactores implícitos del PIB por oferta en paridad de poder adquisitivo $\left(\mathrm{PPP} / \mathrm{XR}\right.$ ) con la variable $p l_{\text {gdpo }}$; para el grupo de países de la Unión Europea se consideró como economía líder Alemania, por lo que su PIB se tomó el ingreso externo en las funciones de exportación y el deflactor implícito de su PIB como los precios externos para el cálculo de los precios relativos; y, para el caso de los países de América Latina se utilizó como economía líder a Estados Unidos (Feenstra, Inklaar, \& Timmer, 2015). Todas

\footnotetext{
1 En el marco analítico de Thirlwall y en muchos de los trabajos aplicados el supuesto de cuenta corriente equilibrada se mantiene y no se analiza la posibilidad de los desequilibrios de balanza de pagos de los países previos a la integración apertura comercial.
} 
las variables que se utilizan se definen a precios constantes de paridad de compra de 2005. Las variables generadas tienen información completa suficiente para estimar modelos panel balanceado para cada uno de los sub periodos; 19571968 y 19691992 para la Unión Europea y 19801994 y 19952012 para América Latina.

\subsection{Especificación econométrica}

Para analizar y evaluar la importancia de la integración económica y la apertura comercial en el crecimiento económico, se utilizan los resultados del enfoque comparativo, donde las modificaciones más importantes se manifestaron en periodos históricos específicos en ambas regiones; en el periodo 19571992 para Europa y 1980-2007 para América Latina. Por un lado el proceso inicial de preferencias arancelarias en Europa comenzó alrededor de 1957, fecha en que entró en vigor la Comunidad Económica Europea, su reforzamiento hacia 1968 y su completitud en 1992 con la conformación de lo que hoy conocemos como "Unión Europea". Para la construcción de los modelos econométricos se eligieron los países de acuerdo con el proceso de integración inicial en la Unión Europea: Austria, Bélgica, España, Finlandia, Francia, Irlanda, Italia, Luxemburgo, Holanda y Portugal. ${ }^{2}$ Para América Latina el momento histórico relevante fue 1994, se considera tomando la transformación de la ALALC en ALADI en 1980 y su ruptura en el G3 (México, Colombia y Venezuela) en 1994 y en el MERCOSUR en 1991 consolidado hacia 1994 con la propuesta de la creación de la ALCSA y por supuesto de la entrada en vigor del TLCAN en 1994. Para el caso latinoamericano, ${ }^{3}$ se consideraron los siguientes países: Argentina (ALADI y MERCOSUR), Bolivia (ALADI), Brasil (ALADI y MERCOSUR), Chile (ALADI), México (ALADI, TLCAN y G3), Paraguay (ALADI y MERCOSUR), Perú (ALADI), Uruguay (ALADI y MERCOSUR) y Venezuela (ALADI y G 3). Con lo anterior, se plantean los sub periodos del antes y del después de la integración económica o apertura comercial de 19571968 y 19691992 para la Unión Europea y 19801994 y 19952007 para América Latina. Con la estimaciones de los modelos de exportaciones e importaciones por país, se obtendrán los parámetros y elasticidades relevantes para cada sub periodo y se definirá la existencia de cambios estructurales con los cuales se puede concluir el papel de la integración económica y la apertura comercial sobre el crecimiento económico con restricciones de balanza de pagos que plantea la hipótesis de Thirlwall versus la de Harrod.

Para probar las hipótesis se estimaron de manera independiente las funciones de exportaciones y de importaciones (Thirlwall \& Pacheco, 2006) y de ellas se obtuvieron las elasticidades precios relativos e ingreso para cada sub periodo y se sustituyeron en la ecuación de crecimiento en su forma reducida compatible con la balanza de pagos para cada grupo de países de las regiones.

\subsubsection{Función de exportaciones}

El crecimiento de las exportaciones medidas por el diferencial $(\Delta)$ del logaritmo $\left(X_{i t}\right)$, se determinan por una especificación clásica de

\footnotetext{
2 En el grupo no se incluyó a Alemania, debido a que se incorpora como economía líder en el análisis del comercio exterior.

3 Se excluye Colombia (perteneciente al G3 y a la ALADI) y Ecuador (miembro de la ALADI)
} 
panel con efectos individuales (fijos o aleatorios) en el crecimiento individual independiente $\left(\beta_{(0, i)}\right)$ y de los crecimientos de los precios relativos $\Delta \ln P r_{i t}=$ $\Delta \ln \left(p_{f, i t} * e_{, i t} / p_{d, i t}\right)$ y el ingreso externo $\left(\Delta \ln Y_{i} t^{*}\right)$. Que para el caso de la Unión Europea, los $i$ - diez países fueron Austria, Bélgica, España, Finlandia, Francia, Irlanda, Italia, Luxemburgo, Holanda y Portugal; el ingreso externo o del resto del mundo fue el PIB de Alemania $\left(Y_{l t}^{*}\right)$; y, los precios relativos se calcularon a precios de paridad de compra de 2005. Para América Latina, los $i$ - nueve países fueron Argentina, Brasil, Bolivia, Chile, México, Paraguay, Perú, Uruguay y Venezuela; el ingreso externo o del resto del mundo fue el PIB de Estados Unidos; y, los precios relativos también se calcularon a precios de paridad de compra de 2005. Los signos esperados de las elasticidades precios relativos e ingreso externo son $\eta_{i}>0$ y $\varepsilon_{i}>0$ respectivamente; ver ecuación 5 .

$$
\Delta \ln X_{\mathrm{it}}=\beta_{0, \mathrm{i}}+\eta_{i} \Delta \ln P r_{i t}+\varepsilon_{i} \Delta \ln Y_{i t}^{*}+\mathrm{ux}_{\mathrm{it}}
$$

\subsubsection{Función de importaciones}

La función de crecimiento de las importaciones $\left(\Delta \ln M_{\mathrm{it}}\right)$ también se determinan por una especificación con efectos individuales (fijos o aleatorios) en el crecimiento individual independiente $\left(\alpha_{0, \mathrm{i}}\right)$, por el los crecimientos de los precios relativos $\Delta l n P r_{i t}$ y del ingreso domestico medido con el PIB de los países $\Delta \ln Y_{\text {it }}$. Los signos esperados de las elasticidades precios relativos e ingreso nacional son $\psi_{i}<0$ y $\pi_{i}>0$ respectivamente; ver ecuación 6 .

$$
\Delta \ln M_{\mathrm{it}}=\alpha_{0, \mathrm{i}}+\psi_{i} \Delta \ln P r_{i t}+\pi_{i} \Delta \ln Y_{\mathrm{it}}+\mathrm{um}_{\mathrm{it}}
$$

\subsection{Análisis de integración y cointegración}

El análisis de integración y cointegración en panel es el procedimiento econométrico que se utilizó para garantizar relaciones económicas de largo plazo en los modelos de exportaciones e importaciones. Para el análisis de integración se utilizaron las pruebas de Levin, Lin y Chu (2002) e Im, Pesaran y Shin (2003), que se pueden especificar de acuerdo a la ecuación 7. En el caso de Levin-Lin-Chu (LLC), la prueba de hipótesis nula es que todos los elementos de sección cruzada tiene la misma raíz unitaria $H_{0}=\phi_{i}=\phi=0$, con respecto a la hipótesis alternativa de estacionario $H_{0}=\phi_{i}=\phi<0$; ellos, proponen un estadístico $t$ ajustado $\left(t_{\delta}^{*}\right)$ para probar las hipótesis. Mientras que en el caso de la prueba de Im-Pesaran-Shin (IPS), el supuesto de raíz unitaria común desaparece y se asumen raíces unitarias individuales en las pruebas de hipótesis; ahora $H_{0}=\phi_{i}=0$ versus la hipótesis alternativa de estacionariedad individual $H_{0}=\phi_{i}<0$. Los estadísticos para las pruebas IPS son variados y por tanto la especificación de la ecuación 7 puede variar, para tener un análisis equivalente con la prueba LLC se utiliza el estadístico $W_{t-b a r}$ que incluye rezagos en la ecuación 7 ; que es la parte $\sum_{j=1}^{p} \theta_{\mathrm{ij}} \Delta y_{\mathrm{i}, \mathrm{t}-\mathrm{j}}$.

$$
\Delta y_{i t}=\phi_{i} y_{\mathrm{i}, \mathrm{t}-1}+Z_{i t}^{\prime} \gamma_{i}+\sum_{j=1}^{p} \theta_{i j} \Delta y_{i, t-j}+\varepsilon_{i t}
$$

Mientras que para el análisis de cointegración de las variables que se definieron en los modelos de exportaciones e importaciones, se utiliza la metodología de 
Westerlund (2007) y Persyn-Westerlund (2008). El procedimiento de Westerlund y Persyn-Westerlund consiste en cuatro pruebas en panel que se construyen con base en la estimación de modelos de corrección de error (mce), que considera la heterogeneidad en la relación de largo plazo como en la dinámica de corto plazo y cierto grado de dependencia contemporánea (entre los elementos de la sección cruzada). Esta dependencia contemporánea es incorporada en la metodología de Westerlund (2007) y Persyn-Westerlund (2008) por medio de una aproximación bootstrap siguiendo el método sugerido por Chang (2004).

Para definir las cuatro pruebas, la especificación del modelo de corrección de error en panel es la siguiente:

$$
\Delta y_{\mathrm{it}}=\delta_{i}^{\prime} d_{t}+\alpha_{\mathrm{i}} y_{\mathrm{i}, \mathrm{t}-1}+\lambda_{i}^{\prime} x_{\mathrm{i}, \mathrm{t}-1}+\sum_{j=1}^{p_{i}} \alpha_{\mathrm{ij}} \Delta y_{\mathrm{i}, \mathrm{t}-\mathrm{j}}+\sum_{j=-q}^{p_{i}} \gamma_{\mathrm{ij}} \Delta x_{\mathrm{i}, \mathrm{t}-\mathrm{j}}+\mathrm{e}_{\mathrm{it}}
$$

Mientras que para el análisis de cointegración de las variables que se definieron en los modelos de exportaciones e importaciones, se utiliza la metodología de Westerlund (2007) y Persyn-Westerlund (2008). El procedimiento de Westerlund y Persyn-Westerlund consiste en cuatro pruebas en panel que se construyen con base en la estimación de modelos de corrección de error (mce), que considera la heterogeneidad en la relación de largo plazo como en la dinámica de corto plazo y cierto grado de dependencia contemporánea (entre los elementos de la sección cruzada). Esta dependencia contemporánea es incorporada en la metodología de Westerlund (2007) y Persyn-Westerlund (2008) por medio de una aproximación bootstrap siguiendo el método sugerido por Chang (2004).

Para definir las cuatro pruebas, la especificación del modelo de corrección de error en panel es la siguiente:

$$
\Delta y_{\mathrm{it}}=\delta_{i}^{\prime} d_{t}+\alpha_{\mathrm{i}} y_{\mathrm{i}, \mathrm{t}-1}+\lambda_{i}^{\prime} x_{\mathrm{i}, \mathrm{t}-1}+\sum_{j=1}^{p_{i}} \alpha_{\mathrm{ij}} \Delta y_{\mathrm{i}, \mathrm{t}-\mathrm{j}}+\sum_{j=-q}^{p_{i}} \gamma_{\mathrm{ij}} \Delta x_{\mathrm{i}, \mathrm{t}-\mathrm{j}}+\mathrm{e}_{\mathrm{it}}
$$

Donde $\lambda_{i}^{\prime}=-\alpha_{\mathrm{i}} \beta_{i}^{\prime}$, el parámetro $\alpha_{i}$ determina la velocidad en la cual la relación de corto plazo se ajusta hacia el equilibrio de largo plazo $\left(y_{\mathrm{i}, \mathrm{t}-1}-\beta_{i}^{\prime} x_{\mathrm{i}, \mathrm{t}-1}\right)$, con el vector de parámetros $\beta_{i}^{\prime}$ de largo plazo. Con ello, se plantea que $y_{i t}$ y $x_{(i, t)}$ están cointegrados si se cumple que $\alpha_{i}<0$ y en términos absolutos menor que uno, pero si $\alpha_{i}=0$ entonces no están cointegrados. En tal sentido la hipótesis nula de no cointegración se escribe como $H_{0}: \alpha_{i}=0$ para todos los $i$ y para probar cointegración se proponen dos grupos de pruebas hipótesis alternativas (Westerlund, 2007; Persyn-Westerlund, 2008): 1) Las pruebas de grupos de medias, que no requieren que los $\alpha_{i}$ s sean iguales, pero la hipótesis nula es definida con respecto a la alternativa $H_{1}^{g}: \alpha_{i}<0$ para al menos uno de los $i$

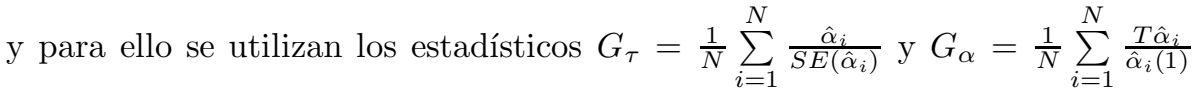
donde $\hat{\alpha}_{i}$ y $S E\left(\hat{\alpha}_{i}\right)$ son la media y su error estándar; y, 2) La pruebas de panel, que asume que los $\alpha_{i}$ son iguales para todos los $i$, por lo que la hipótesis nula se prueba versus la alternativa $H_{1}^{p}: \alpha_{\mathrm{i}}=\alpha<0$ para todo $i$, con los estadísticos 
$P_{\tau}=\frac{\hat{\alpha}_{i}}{S E\left(\hat{\alpha}_{i}\right)}$ y $P_{\alpha}=T \hat{\alpha}_{i}$. Las pruebas de hipótesis tienen tres posibilidades dependiendo de incluir o no la constante y la tendencia determinística en la ecuación 7, las cuales definen como: 1) Sin tendencia determinística, que se obtiene cuando se supone que $d_{t=0} ; 2$ ) Con constante, que se define cuando $\left.d_{t=1} ; \mathrm{y}, 3\right)$ Con constante y tendencia cuando $d_{t=(1, t)}$.

4. Resultados de las estimaciones y cálculo del crecimiento potencial con restricciones de Balanza de Pagos

\subsection{Resultados del análisis de integración}

Para el análisis de integración se aplicaron las pruebas de raíz unitaria LLC e IPS en el logaritmo natural del nivel y su primera diferencia, para cada una de las variables de las funciones de exportaciones e importaciones en las dos regiones y en los periodos específicos; de 1957-1992 para los países de la Unión Europea y de 1980-2011 para los de América Latina (ver tabla 1).

Los resultados de las pruebas LLC e IPS para las variables $\ln X_{i t}, \ln Y_{i t} * \mathrm{y}$ $\ln \mathrm{Pr}_{i t}$ de la función de exportaciones de la Unión Europea, muestran que las exportaciones y los precios relativos son I(1) y el ingreso externo (Alemania) es de I(0). En el caso de las variables de las exportaciones de América Latina, también se encontró que las exportaciones y los precios relativos son I(1), pero el ingreso externo (Estados Unidos) es $\mathrm{I}(0)$, cuando se supone raíz unitaria conjunta (prueba LLC), e I(1) si se supone raíz unitaria individual (IPS). En el caso de las variables $\ln M_{i t}, \Delta \ln Y_{i t}$ y $\ln P r_{i t}$ para la función de importaciones de la Unión Europea, se encontró que las importaciones y los precios relativos son I(1), el ingreso interno es I(0) con la prueba LLC e I(1) con la prueba IPS. En tanto, las tres variables de la función de importaciones del grupo de países de américa Latina son I(1).

Lo importante de estos resultados, es que en los casos donde se tienen dos variables $\mathrm{I}(1)$ y una $\mathrm{I}(0)$ en las funciones de exportaciones e importaciones, aumenta la posibilidad de encontrar un vector cointegración.

Tabla 1: Prueba de raíz unitaria en panel para variables de las funciones exportaciones e importaciones de países de la Unión Europea (1957-1992)

y América Latina (1980-2011)

\begin{tabular}{|c|c|c|c|c|c|}
\hline \multicolumn{6}{|l|}{ Países de Unión Europea } \\
\hline Variables & $\ln X$ & $\ln Y^{*}$ & $\ln M$ & $\ln Y$ & $\ln P r$ \\
\hline $\begin{array}{l}\text { Pruebas estadisticas } \\
\text { Levin-Lin-Chu }\end{array}$ & $p$-value & $p$-value & p-value & $p$-value & $p$-value \\
\hline $\begin{array}{l}\mathrm{t}^{*} \text { _ajustada } \\
\text { Im-Pesaran Shin }\end{array}$ & 0.471 & 0.000 & 0.295 & 0.000 & 0.083 \\
\hline W-t-bar & 1.000 & 0.000 & 1.000 & 0.496 & 0.125 \\
\hline Variables en diferencias & D. $\ln X$ & $D \cdot \ln Y^{*}$ & D. $\ln M$ & D. $\ln Y$ & D. $\ln P r$ \\
\hline $\begin{array}{l}\text { Pruebas estadisticas } \\
\text { Levin-Lin-Chu }\end{array}$ & $p$-value & $p$-value & $p$-value & $p$-value & $p$-value \\
\hline $\begin{array}{l}\text { t*_ajustada }^{\text {Im-Pesaran_Shin }} \\
\text { Im }\end{array}$ & 0.000 & 0.000 & 0.000 & 0.000 & 0.000 \\
\hline$\overline{\mathrm{W}}$-t-bar & 0.000 & 0.000 & 0.000 & 0.000 & 0.000 \\
\hline
\end{tabular}


Tabla 1: Prueba de raíz unitaria en panel para variables de las funciones exportaciones e importaciones de países de la Unión Europea (1957-1992)

y América Latina (1980-2011)

\begin{tabular}{|c|c|c|c|c|c|}
\hline \multicolumn{6}{|l|}{ Países de América Latina } \\
\hline Variables & $\ln X$ & $\ln Y^{*}$ & $\ln M$ & $\ln Y$ & $\ln P r$ \\
\hline $\begin{array}{l}\text { Pruebas estadísticas } \\
\text { Levin-Lin-Chu }\end{array}$ & p-value & $p$-value & p-value & $p$-value & $p$-value \\
\hline $\begin{array}{l}{ }^{t^{*}} \text { ajustada } \\
\text { Im-Pesaran Shin }\end{array}$ & 0.958 & 0.000 & 0.996 & 0.999 & 0.064 \\
\hline W-t-bar & 1.000 & 0.969 & 1.000 & 1.000 & 0.062 \\
\hline Variables en diferencias & D. $\ln X$ & $D \cdot \ln Y^{*}$ & D. $\ln M$ & D. $\ln Y$ & D. $\ln P r$ \\
\hline $\begin{array}{l}\text { Pruebas estadísticas } \\
\text { Levin-Lin-Chu }\end{array}$ & p-value & p-value & p-value & $p$-value & $p$-value \\
\hline $\begin{array}{l}t^{*} \text { _ajustada } \\
\text { Im-Pesaran_Shin }\end{array}$ & 0.000 & 0.000 & 0.000 & 0.000 & 0.001 \\
\hline W-t-bar & 0.000 & 0.000 & 0.000 & 0.000 & 0.000 \\
\hline
\end{tabular}

Notas: Todas las pruebas se elaboraron con constante y sin tendencia; en la prueba LLC se utilizó el kernel Bartlet con algoritmo Newey-West.

\subsection{Resultados del análisis de cointegración}

Las pruebas de cointegración en panel de Westerlund (2007) y Persyn-Westerlund (2008) se aplicaron a las variables de las exportaciones, precios relativos e ingreso externo y a las variables de importaciones, precios relativos e ingreso interno, también se analiza la posibilidad de que la relación de largo plazo se sostenga solamente con las variables de ingreso externo e interno en las funciones de exportación e importación respectivamente, esto con la idea de dar indicios sobre las hipótesis de Thirlwall versus la de Harrod. En la tabla 2 se presentan los resultados de las pruebas aplicadas en las funciones de exportación e importación para los países de la Unión Europea. Para el caso de la relación entre las exportaciones, los precios relativos y el ingreso externo, los resultados muestran que existe la posibilidad de que estén cointegradas al suponer tanto heterogeneidad como homogeneidad de la relación de largo plazo cuando se considera la constante y tendencia heterogénea, la relación es débil cuando la constante es heterogénea, pero sin constante y tendencia no existe evidencia de cointegración. Para analizar el caso de que solamente estén cointegrados las exportaciones y el ingreso externo y por tanto el planteamiento de Harrod sea el adecuado, se aplican las pruebas sin considerar los precios relativos. Los resultados anteriores se mantienen y se vuelven más robustos para el caso de una constante heterogénea. En la segunda parte de la tabla 2, se presentan los resultados para la relación entre importaciones, precios relativos e ingreso interno. En este caso los resultados más robustos son cuando se considera únicamente la constante heterogénea y en segundo lugar con constante y tendencia, como en el caso de la función de exportaciones no hay evidencias de cointegración cuando no se consideran la constante y tendencia heterogénea. En el caso de la función de importaciones sin precios relativos, los resultados se mantienen y mejoran para el caso de cointegración con constante y tendencia. 
Tabla 2. Prueba de cointegración en panel de Westerlund (2007) y Persyn-Westerlund (2008) para indicadores de países de la Unión Europea

\begin{tabular}{|c|c|c|c|c|c|c|}
\hline \multicolumn{7}{|c|}{ Función de exportaciones } \\
\hline & \multicolumn{3}{|c|}{ Con precios relativos e ingreso externo } & \multicolumn{3}{|c|}{ Con restricción de precios } \\
\hline \multirow[t]{2}{*}{ Estadístico } & Constante y tendencia & Constonte & $\begin{array}{c}\text { sin constantey } \\
\text { tendencia }\end{array}$ & $\begin{array}{c}\text { Constantey } \\
\text { tendencio }\end{array}$ & Constonte & $\begin{array}{c}\text { sin constante y } \\
\text { tendencia }\end{array}$ \\
\hline & p-value & p-value & p-value & p-value & p-volue & p-value \\
\hline Gt & 0.000 & 0.060 & 0.784 & 0.049 & 0.000 & 0.307 \\
\hline Ga & 0.695 & 0.469 & 0.967 & 0.823 & 0.070 & 0.783 \\
\hline Pt & 0.000 & 0.737 & 0.583 & 0.026 & 0.199 & 0.148 \\
\hline $\mathrm{Pa}$ & 0.129 & 0.646 & 0.585 & 0.333 & 0.090 & 0.098 \\
\hline \multicolumn{7}{|c|}{ Función de importaciones } \\
\hline & \multicolumn{2}{|c|}{ Con precios relativos e ingreso interno } & \multicolumn{3}{|c|}{ Con restricción de precios } & \\
\hline \multirow[t]{2}{*}{ Estadístico } & Constonte y tendencia & Constonte & $\begin{array}{c}\text { sin constantey } \\
\text { tendencia }\end{array}$ & $\begin{array}{c}\text { Constantey } \\
\text { tendencia }\end{array}$ & Constonte & $\begin{array}{c}\text { sin constantey } \\
\text { tendencia }\end{array}$ \\
\hline & p-value & p-value & p-value & p-value & p-value & p-value \\
\hline Gt & 0.000 & 0.000 & 0.926 & 0.000 & 0.001 & 0.997 \\
\hline Ga & 0.111 & 0.000 & 0.972 & 0.098 & 0.000 & 0.979 \\
\hline Pt & 0.021 & 0.001 & 0.564 & 0.001 & 0.000 & 0.439 \\
\hline $\mathrm{Pa}$ & 0.305 & 0.000 & 0.607 & 0.233 & 0.000 & 0.412 \\
\hline
\end{tabular}

Nota: En las pruebas se utilizaron un rezago en la parte endógena y exógena en mce

Para el caso de la función de exportaciones en países de América Latina, los resultados que se muestran en la tabla 3 indican que existe una relación débil de largo plazo cuando se considera la constante heterogénea y de no cointegración con y sin la combinación constante y tendencia. Pero cuando se analizan las exportaciones y el ingreso sin los precios relativos, la posibilidad de cointegración aumenta para el caso de parámetros de ajuste de corrección de error iguales y constantes heterogéneas. Para la relación de importaciones, precios relativos e ingreso interno, existe evidencia débil de cointegración con y sin la combinación de constante y tendencia, y mayor posibilidad cuando se considera la constante heterogénea tanto para el caso de parámetro de corrección de error diferente como igual entre los países. Cuando se consideran únicamente las importaciones y el ingreso externo, los estadísticos basados en t, para los tres casos de constante y tendencia, constante y sin constante y tendencia muestran mayores posibilidades de existencia de cointegración. 
Tabla 3: Prueba de cointegración en panel de Westerlund (2007) y Persyn-Westerlund (2008) para indicadores de países de América Latina

\begin{tabular}{|c|c|c|c|c|c|c|}
\hline \multicolumn{7}{|c|}{ Función de exportaciones } \\
\hline & \multicolumn{2}{|c|}{ Con precios relativos e ingreso externo } & \multicolumn{3}{|c|}{ Con restricción de precios } & \\
\hline Estadistico & $\begin{array}{c}\text { Constantey } \\
\text { tendencia }\end{array}$ & Constonte & $\begin{array}{c}\text { sin constantey } \\
\text { tendencia }\end{array}$ & $\begin{array}{l}\text { Constantey } \\
\text { tendencia }\end{array}$ & Constante & $\begin{array}{c}\text { sin constantey } \\
\text { tendencia }\end{array}$ \\
\hline & $p$-volue & p-volue & $p$-volue & p-volue & p-volue & p-volue \\
\hline Gt & 0.681 & 0.183 & 0.368 & 0.790 & 0.200 & 0.672 \\
\hline Ga & 0.999 & 0.814 & 0.903 & 0.992 & 0.330 & 0.850 \\
\hline Pt & 0.628 & 0.079 & 0.225 & 0.456 & 0.020 & 0.172 \\
\hline $\mathrm{Pa}$ & 0.973 & 0.258 & 0.462 & 0.737 & 0.002 & 0.218 \\
\hline \multicolumn{7}{|c|}{ Función de importaciones } \\
\hline & \multicolumn{2}{|c|}{ Con precios relativos e ingreso interno } & \multicolumn{3}{|c|}{ Con restricción de precios } & \\
\hline Estadístico & $\begin{array}{c}\text { Constantey } \\
\text { tendencia }\end{array}$ & Constonte & $\begin{array}{c}\text { sin constante y } \\
\text { tendencia }\end{array}$ & $\begin{array}{l}\text { Constantey } \\
\text { tendencia }\end{array}$ & Constante & $\begin{array}{c}\text { sin constantey } \\
\text { tendencia }\end{array}$ \\
\hline & $p$-volue & p-volue & $p$-value & $p$-volue & p-volue & p-value \\
\hline Gt & 0.036 & 0.051 & 0.215 & 0.042 & 0.000 & 0.058 \\
\hline Ga & 0.988 & 0.578 & 0.891 & 0.950 & 0.217 & 0.642 \\
\hline Pt & 0.113 & 0.020 & 0.060 & 0.097 & 0.001 & 0.051 \\
\hline $\mathrm{Pa}$ & 0.918 & 0.210 & 0.358 & 0.872 & 0.030 & 0.244 \\
\hline
\end{tabular}

Nota: En las pruebas se utilizaron un rezago en la parte endógena y exógena en mce

\subsection{Análisis de resultados de modelos panel log-log}

La estimación de las elasticidades precio relativo e ingreso para la funciones de exportaciones e importaciones, se pueden obtener al estimar las especificaciones 5 y 6 pero sin el diferencial Deta y lo que se obtiene son las elasticidades de largo plazo compatibles con el concepto de cointegración. En la tabla 4 se presentan estas estimaciones de panel log-log con efectos aleatorios de las funciones de exportaciones e importaciones para los países de la Unión Europea. Los resultados para el periodo previo a la integración (19571968) muestran una elasticidad precio negativa y no significativa para las exportaciones y una elasticidad ingreso externo positiva y significativa de 1.78. Para confirmar que las exportaciones en este periodo se pueden explicar solamente por el ingreso externo, se estimó la función sin precios relativos y la elasticidad ingreso (1.73) no es muy diferente a la versión con precios relativos. En el segundo periodo (1969-1992) identificado como de integración para los países de la unión europea, la elasticidad precio relativos de las exportaciones se mantuvo con el signo negativo, y aunque es muy pequeña ahora es significativa. Por otro lado, los resultados de la elasticidad ingreso externo (2.54) muestran que las exportaciones se hicieron más sensibles al crecimiento 
económico de la economía líder con respecto al primer periodo. El resultado del modelo panel sin precios relativos, confirma que esta variable se puede eliminar y que la elasticidad ingreso externo es mayor para el periodo de integración económica.

Para el caso de las importaciones de la Unión Europea, que también se presentan en la tabla 4, la elasticidad precios relativos es pequeña, negativa pero no significativa y una elasticidad ingreso nacional de 1.50 para el primer periodo. Este resultado es más robusto desde el punto de vista econométrico, cuando se estima el modelo de importaciones sin considerar los precios relativos, aunque la elasticidad ingreso de 1.44 resultó menor al modelo con precios relativos. Para el periodo de integración económica, la elasticidad precios relativos (-0.63) es negativa y mayor, y la elasticidad ingreso nacional (1.57) aumenta ligeramente con respecto al primer periodo. Con la eliminación de los precios relativos en la función de importaciones, la elasticidad ingreso de 1.69 indica mayor sensibilidad al comportamiento de la economía nacional.

En la tabla 5 se presentan los resultados de las estimaciones panel log-log para los países de América Latina. Para el periodo previo a los acuerdos comerciales (1980-1994) se obtuvieron para las exportaciones una elasticidad precio positiva (0.06), muy pequeña pero no significativa y una sensibilidad ingreso externo positiva, inelástica y significativa (0.50). Del ejercicio de no tomar en cuenta los precios relativos, los resultados confirman que las exportaciones en este periodo se pueden explicar solamente por la elasticidad ingreso externo (0.55). Mientras que para el periodo de acuerdos comerciales (1995-2011) la elasticidad precios relativos de las exportaciones es ahora con signo negativo y es significativa, el cambio más importante es que las exportaciones se volvieron más sensibles al ingreso externo (2.86). Del ejercicio sin precios relativos, se confirma que las exportaciones se pueden explicar principalmente con la elasticidad ingreso externo.

Las estimaciones para las importaciones de los países de América Latina para el primer periodo, muestran inelasticidades en precios relativos y el ingreso nacional significativas; en primer caso de -0.38 y 0.90 . No obstante, de que la elasticidad precio relativos tiene el signo correcto y es significativo, el ejercicio con restricción de precios relativos muestra que las importaciones se pueden explicar solamente con el ingreso nacional (0.88). Con la apertura comercial, las importaciones de América Latina mantienen su inelasticidad precios relativos $(-0.48)$ y se vuelven elásticas al ingreso nacional (1.36). De la misma manera que en el primer periodo, el ejercicio de restricción de precios relativos confirma que las importaciones se pueden explicar robustamente con el ingreso nacional (1.39). 
42 Nueva Época REMEF (The Mexican Journal of Economics and Finance)

Tabla 4: Modelos panel de exportaciones e importaciones en países de la Unión Europea

\begin{tabular}{|c|c|c|c|c|c|c|c|c|}
\hline \multirow[b]{3}{*}{ Variable explicativa } & \multicolumn{8}{|c|}{ Función de exportaciones } \\
\hline & \multicolumn{4}{|c|}{ Primer periodo: $1957-1968$} & \multicolumn{4}{|c|}{ Segundo periodo: $1969-1992$} \\
\hline & $\log -\log$ & $\begin{array}{l}\text { Intervalo } \\
\text { con } 95 \% \text { de } \\
\text { conf. }\end{array}$ & Incrementos & $\begin{array}{l}\text { Intervalo } \\
\text { con } 95 \% \text { de } \\
\text { conf. }\end{array}$ & $\log -\log$ & $\begin{array}{l}\text { Intervalo } \\
\text { con } 95 \% \text { de } \\
\text { conf. }\end{array}$ & Incrementos & $\begin{array}{c}\text { Intervalo } \\
\text { con } 95 \% \text { de } \\
\text { conf. }\end{array}$ \\
\hline \multicolumn{9}{|l|}{ Elasticidades } \\
\hline Precios relativos & $\begin{array}{l}-0.25 \\
(0.30)\end{array}$ & {$[-0.71,0.216]$} & $\begin{array}{c}0.17 \\
(0.38)\end{array}$ & {$[-0.21,0.55]$} & $\begin{array}{l}-0.38 \\
(0.00)\end{array}$ & {$[-0.59,-0.17]$} & $\begin{array}{c}0.24 \\
(0.04)\end{array}$ & {$[0.014,0.46]$} \\
\hline Ingreso externo & $\begin{array}{c}1.78 \\
(0.00)\end{array}$ & {$[1.56,1.99]$} & $\begin{array}{c}0.68 \\
(0.13)\end{array}$ & {$[-0.18,1.54]$} & $\begin{array}{c}2.54 \\
(0.00)\end{array}$ & {$[2.42,2.65]$} & $\begin{array}{c}0.57 \\
(0.08)\end{array}$ & {$[-0.07,1.21]$} \\
\hline R2 (overall) & 0.07 & & 0.03 & & 0.13 & & 0.04 & \\
\hline \multicolumn{9}{|l|}{ Con restricción de precios } \\
\hline Ingreso externo & $\begin{array}{c}1.73 \\
(0.00)\end{array}$ & {$[1.54,1.91]$} & $\begin{array}{c}0.74 \\
(0.09)\end{array}$ & {$[-0.12,1.59]$} & $\begin{array}{c}2.62 \\
(0.00)\end{array}$ & {$[2.50,2.72]$} & $\begin{array}{c}0.70 \\
(0.03)\end{array}$ & {$[0.061,1.34]$} \\
\hline \multirow[t]{3}{*}{ R2 (overall) } & 0.06 & & 0.02 & & 0.126 & & 0.019 & \\
\hline & \multicolumn{8}{|c|}{ Función de importaciones } \\
\hline & \multicolumn{4}{|c|}{ Primer periodo: $1957-1968$} & \multicolumn{4}{|c|}{ Segundo periodo: $1969-1992$} \\
\hline Variable explicativa & $\log -\log$ & $\begin{array}{l}\text { Intervalo } \\
\text { con } 95 \% \text { de } \\
\text { conf. }\end{array}$ & Incrementos & $\begin{array}{l}\text { Intervalo } \\
\text { con } 95 \% \text { de } \\
\text { conf. }\end{array}$ & $\log -\log$ & $\begin{array}{l}\text { Intervalo } \\
\text { con } 95 \% \text { de } \\
\text { conf. }\end{array}$ & Incrementos & $\begin{array}{c}\text { Intervalo } \\
\text { con } 95 \% \text { de } \\
\text { conf. }\end{array}$ \\
\hline \multicolumn{9}{|l|}{ Elasticidades } \\
\hline Precios relativos & $\begin{array}{l}-0.35 \\
(0.13)\end{array}$ & {$[-0.79,0.104]$} & $\begin{array}{l}-0.63 \\
(0.00)\end{array}$ & {$[-0.99,-0.2]$} & $\begin{array}{l}-0.63 \\
(0.00)\end{array}$ & {$[-0.83,-0.42]$} & $\begin{array}{l}-0.17 \\
(0.18)\end{array}$ & {$[-0.41,0.07]$} \\
\hline Ingreso nacional & $\begin{array}{c}1.50 \\
(0.00)\end{array}$ & {$[1.33,1.66]$} & $\begin{array}{c}1.98 \\
(0.00)\end{array}$ & {$[1.29,2.65]$} & $\begin{array}{c}1.57 \\
(0.00)\end{array}$ & {$[1.48,1.65]$} & $\begin{array}{c}1.59 \\
(0.00)\end{array}$ & {$[1.09,2.08]$} \\
\hline $\begin{array}{l}\text { R2 (overall) } \\
\text { Con restricción de precic }\end{array}$ & 0.07 & & 0.03 & & 0.13 & & 0.04 & \\
\hline Ingreso nacional & $\begin{array}{c}1.44 \\
(0.00)\end{array}$ & {$[1.28,1.59]$} & $\begin{array}{c}2.03 \\
(0.00)\end{array}$ & {$[1.31,2.73]$} & $\begin{array}{c}1.69 \\
(0.00)\end{array}$ & {$[1.60,1.77]$} & $\begin{array}{r}1.52 \\
(0.00)\end{array}$ & {$[1.03,2.00]$} \\
\hline R2 (overall) & 0.73 & & 0.21 & & 0.82 & & 0.138 & \\
\hline
\end{tabular}

Nota: Los modelos panel se estimaron con efectos aleatorios. Entre paréntesis se presenta la probabilidad asociada a la $t$-estadística 
Tabla 5. Modelos panel de exportaciones e importaciones en países de América Latina

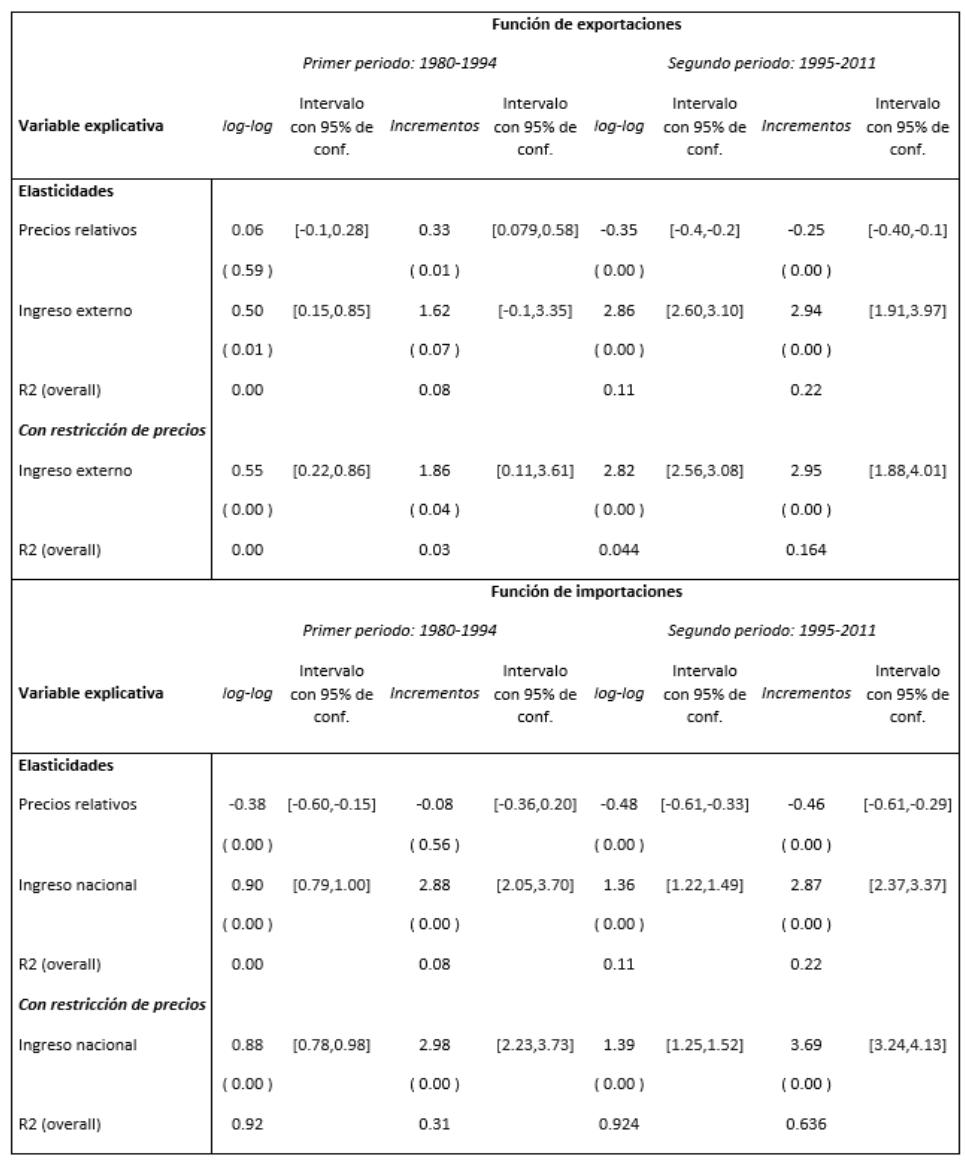

Nota: Los modelos panel se estimaron con efectos aleatorios. Entre paréntesis se presenta la probabilidad asociada a la $t$-estadística

\subsection{Análisis de resultados con modelos de crecimiento}

Con las especificaciones 5 y 6 con crecimientos se obtienen también elasticidades precio relativo e ingreso, que desde el punto de vista del análisis de cointegración representan efectos de corto plazo y se espera que se ajusten hacia las de largo plazo. Los resultados de los modelos panel en diferencias y estimados con efectos aleatorios para las funciones de exportaciones e importaciones de los países de la Unión Europea se presentan en la tabla 4. Para el primer periodo, los resultados son una elasticidad precio positiva, pero de la misma manera que en el modelo log-log, es no significativa y la elasticidad ingreso externo de 0.68 y significativa, y como se esperaba mucho menor a la elasticidad de largo plazo. Con la restricción de los precios relativos, la elasticidad ingreso es muy parecida a la versión con precios relativos pero la significancia se alcanza solamente al 91 por ciento de confianza. En el segundo periodo, los precios relativos son 
significativos y positivos, en contraste con la elasticidad de largo plazo (log-log) que fue negativa y significativa. La elasticidad ingreso es significativa al 92 por ciento de confianza, positiva, menor a la de largo plazo y con respecto al periodo previo a la integración económica. Sin los precios relativos, la elasticidad ingreso externo se volvió más significativa, ligeramente mayor que con precios relativos pero muy parecida a la obtenida en el primer periodo.

Los resultados de la estimación de la función importaciones de los países de la Unión Europea en el primer periodo también se presentan en la tabla 4, donde se encontró que la elasticidad precios relativos de corto plazo es negativa (-0.63) y significativa, mayor a la de largo plazo que resultó no significativa. La elasticidad ingreso para este periodo es significativa y resultó mayor a la de largo plazo y en la versión sin precios relativos, el efecto ingreso se mantuvo prácticamente igual. Para el segundo periodo, los precios relativos son ahora no significativos y la elasticidad muy pequeña, lo más importante en la explicación de las importaciones de corto plazo es el efecto ingreso nacional, con una elasticidad de 1.59 menor a la del primer periodo y muy parecida a la de largo plazo, por lo que el resultado no cambia cuando en la estimación de eliminan los precios relativos.

Los resultados de las estimaciones con incrementos de las exportaciones para los países de América Latina (ver tabla 5), muestran que la elasticidad precios relativos de corto plazo positiva (0.33) y significativa, a diferencia de una elasticidad de largo plazo no significativa y una elasticidad ingreso externo significativa al 93 por ciento de confianza, positiva y mayor a la de largo plazo para el periodo previo a la apertura comercial. De la estimación con restricción de los precios relativos, los resultados muestran una elasticidad ingreso mayor que con precios relativos. Para el segundo periodo, la elasticidad precios relativos tanto de corto como de largo plazo es negativa y significativa, lo cual implica un sesgo con respecto a lo esperado. Mientras que la elasticidad ingreso externo fue de 2.94 significativa, muy parecida a la de largo plazo y mayor a la observada en el periodo previo a la apertura comercial. En este caso, el resultado de la estimación muestra que la elasticidad ingreso externo es muy parecida con y sin precios relativos.

Para el caso de las importaciones de los países de América Latina, la elasticidad precios relativos no es significativa y la elasticidad ingreso nacional de corto plazo es de 2.88, mucho mayor a la de largo plazo de 0.9 y no tan diferente cuando se eliminan los precios relativos en la estimación en el primer periodo. Para el periodo de apertura comercial, la elasticidad precios relativos de corto plazo fue significativa y negativa (-0.46), igual a la de largo plazo pero mayor al periodo previo a la apertura. Y, la elasticidad ingreso nacional fue de 2.87 significativa, mayor que la de largo plazo pero igual al primer periodo y con la restricción de los precios relativos la elasticidad ingreso absorbe toda la explicación de las importaciones y aumenta casi un punto porcentual; de 2.87 a 3.69 .

\subsection{Crecimiento potencial compatible con balanza de pagos}

De acuerdo a los resultados de las estimaciones de los modelos log-log y de crecimiento para las exportaciones e importaciones tanto para los países de la Unión Europea como de América Latina, se puede concluir que el mecanismo de los precios relativos no fue relevante en la explicación de los procesos de 
integración y de apertura comercial respectivamente en cada zona. Desde el punto de vista teórico, el análisis del crecimiento económico compatible con balanza de pagos se tiene que hacer con la ecuación de equilibrio en la versión dinámica del multiplicador del comercio de Harrod (Bairam, 1988; Bairam, 1993; McCombie, 1997; Santos \& Thirlwall, 1999), donde el crecimiento económico es igual a la tasa de crecimiento de las exportaciones entre la elasticidad ingreso interno a importar o en la versión de la elasticidad ingreso externo multiplicada por el crecimiento del ingreso externo entre la elasticidad ingreso interno a importar (Thirlwall, 2003; Thirlwall, 1997). De acuerdo a ello, para analizar el crecimiento económico observado, con el crecimiento potencial compatible con balanza de pagos antes y después de la integración económica en países de la Unión Europea, y de apertura comercial en países de América Latina, se contabilizaron las dos versiones del crecimiento potencial tipo Harrod: 1) Con crecimiento de las exportaciones y elasticidad ingreso a importar $\left.y_{B, i(1)}=x / \pi_{i} ; \mathrm{y}, 2\right)$ Con elasticidad ingreso externo, crecimiento del ingreso externo y elasticidad ingreso a importar $y_{B, i(2)}=\varepsilon\left(y_{l}^{*}\right) / \pi_{i}$. $Y$, de forma complementaria también se contabilizó el crecimiento potencial tipo Thirlwall con los resultados de las elasticidades y crecimientos de los precios relativos $y_{B, i(3)}=\left[\left(1-\left(\eta_{i}+\psi_{i}\right)\right)\left(p_{d, i}-p_{f, i}-e_{i}\right)+\varepsilon\left(y_{l}^{*}\right)\right] / \pi_{i} . \quad$ Los crecimientos potenciales se realizaron con los resultados de las estimaciones de las especificaciones log-log y crecimiento, para comparar los efectos de largo y corto plazo.

La contabilización de los crecimientos observados y los potenciales para los países de la Unión Europea se presentan en la Tabla 6. Lo primero que resalta del crecimiento económico promedio observado $\left(y_{i}\right)$, es que para las mayoría de los países la tasa de crecimiento se redujo del periodo con integración con respecto al primer periodo, con la excepción de Irlanda y Luxemburgo. Las ajustes a la baja del crecimiento económico más importantes entre los dos periodos se presentaron en España, Italia, Francia y Holanda. Para el primer periodo y en comparación con las tasas de crecimiento potenciales de Harrod tipo $2\left(y_{B, i(2)}\right)$ de corto plazo, mantuvieron tasas de crecimiento promedio por arriba del potencial considerando la sensibilidad de sus exportaciones al crecimiento económico de la economía líder (Alemania) y a la propensión a importar. Pero al comparar el crecimiento observado en relación al crecimiento de las exportaciones y la propensión a importar, solamente España, Italia y Holanda mantuvieron un crecimiento económico sin problemas de balanza de pagos. Esto indica que en algunos años mantuvieron problemas de balanza de pagos. Cuando se utilizan los crecimientos potenciales Harrod de largo plazo, los resultados muestran que al considerar cualquiera de los dos tipos de contabilización, la mayoría de los países mantuvo tasas de crecimiento compatible con balanza de pagos, solamente países como Bélgica, España, Francia, Luxemburgo y Portugal mostraron indicios de problemas de crecimiento económico compatible con una balanza de pagos en equilibrio.

Para el periodo considerado de integración económica, los cálculos muestran que los diez países mostrarían problemas de crecimiento económico compatible con la balanza de pagos en el corto plazo, si se comparan con la tasas de crecimiento potencial Harrod tipo 2 y en contraposición la mayoría de ellos no tendría problemas de equilibrio de la balanza de pagos si se compara con 
el crecimiento potencial Harrod tipo 1, con crecimiento de las exportaciones y propensión a importar. En este esquema los países con posibles problemas en el corto plazo, resultaron Luxemburgo y Holanda. En comparación con las tasas de crecimiento de largo plazo Harrod tipo 2 -con elasticidad ingreso externo, crecimiento económico de la economía líder y la propensión a importar- los diez países de la Unión Europea crecieron por debajo del potencial con equilibrio de la balanza de pagos y solamente Luxemburgo y Holanda mantuvieron tasas de crecimiento que al comparar con el potencial Harrod tipo 1, pudieron significar ligeras presiones de balanza de pagos en el largo plazo.

Tabla 6. Crecimiento potencial compatible con balanza de pagos en países de Unión Europea

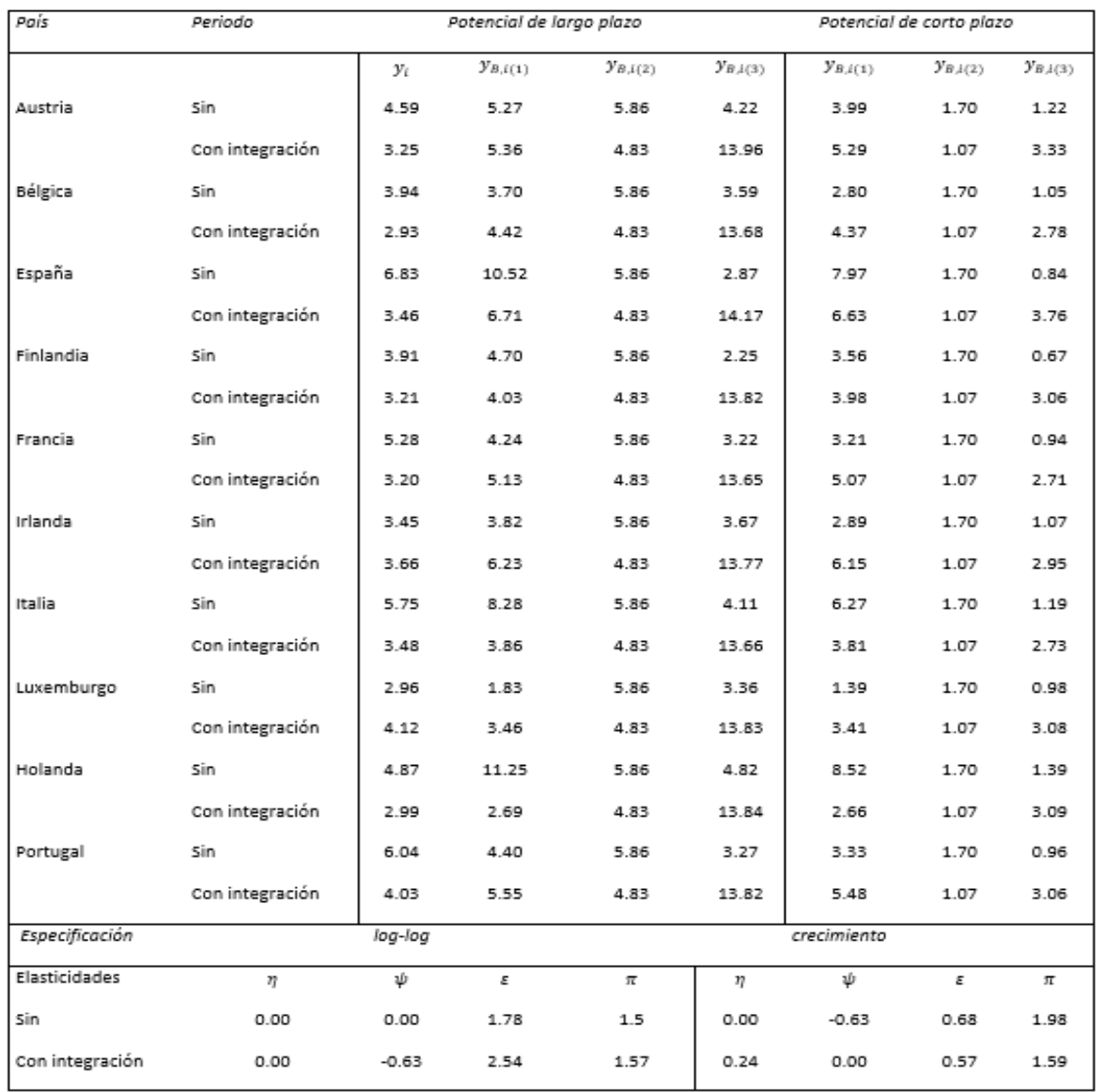

Nota: Elaborado con las elasticidades que se presentan en el cuadro 3, y las tasas de crecimiento económico nacional, de las exportaciones, importaciones, de la economía líder de la región, los precios internos, externos y los tipos de cambios correspondientes. 
Para el caso de América Latina, y en contraposición con los resultados de la Unión Europea, las tasas de crecimiento promedio $\left(y_{i}\right)$ para la mayoría de los países aumentaron en el periodo de apertura comercial, solamente tres países crecieron menos con respeto al periodo de no apertura: Chile, México y Paraguay. Los países con las mayores tasas de crecimiento económico en el periodo de apertura fueron Perú, Bolivia y Argentina. De acuerdo a los crecimientos observados en el periodo previo a la apertura y comparando con las tasas de crecimiento potenciales Harrod tipo $2\left(y_{B, i(2)}\right)$ de corto plazo, donde se considera la sensibilidad de las exportaciones al crecimiento de la economía de Estados Unidos y a la propensión a importar, se encontró que la mayoría de los países crecieron por arriba del potencial, solamente en Bolivia, Perú y Venezuela resultó lo contario. En la comparación con las tasas de crecimiento potenciales de corto plazo Harrod tipo $1\left(y_{B, i(1)}\right)$ - con crecimiento de exportaciones y propensión a importar- los resultados anteriores de crecimiento económico por arriba del potencial y posibles problemas de equilibrio de balanza de pagos se mantienen, pero ahora los países con comportamiento contrario fueron Brasil, México y Venezuela. En comparación con los crecimientos potenciales de largo plazo de los tipos Harrod, los resultados de corto plazo se mantuvieron y confirmaron los posibles problemas de balanza de pagos en países como Argentina, Chile y Paraguay.

Los resultados para el periodo de apertura comercial muestran a los diez países con problemas de crecimiento económico compatible con la balanza de pagos en el corto plazo, cuando se comparan con la tasas de crecimiento potencial Harrod tipo 2; en contraposición, la mayoría de ellos no tendría problemas de equilibrio de la balanza de pagos con el crecimiento potencial Harrod tipo 1; con excepción de Venezuela. Con respecto a las tasas de crecimiento de largo plazo Harrod tipo 1 y 2, la mayoría de los países crecieron por debajo del potencial con equilibrio de la balanza de pagos; nuevamente, con excepción de Venezuela. 
Tabla 7. Crecimiento potencial compatible con balanza de pagos en países de América Latina

\begin{tabular}{|c|c|c|c|c|c|c|c|c|}
\hline \multirow[t]{2}{*}{ País } & \multicolumn{2}{|l|}{ Periodo } & \multicolumn{3}{|c|}{ tencial de largo plazo } & \multicolumn{3}{|c|}{ Potencial de corto plazo } \\
\hline & & $y_{i}$ & $y_{B, i(1)}$ & $y_{B, i(2)}$ & $y_{B, i(3)}$ & $y_{B, i(1)}$ & $y_{B, i(2)}$ & $y_{B, i(3)}$ \\
\hline \multirow[t]{2}{*}{ Argentina } & Sin & 1.62 & -0.07 & 1.57 & 1.52 & -0.05 & 1.59 & 2.07 \\
\hline & Con apertura & 3.82 & 3.93 & 5.12 & 3.59 & 3.88 & 2.49 & 1.79 \\
\hline \multirow[t]{2}{*}{ Bolivia } & Sin & 1.10 & -1.33 & 1.57 & 0.36 & -1.01 & 1.59 & 1.68 \\
\hline & Con apertura & 3.86 & 4.82 & 5.12 & 5.30 & 4.76 & 2.49 & 2.53 \\
\hline \multirow[t]{2}{*}{ Brasil } & Sin & 2.48 & 4.53 & 1.57 & 2.19 & 3.44 & 1.59 & 2.30 \\
\hline & Con apertura & 3.15 & 4.54 & 5.12 & 7.51 & 4.48 & 2.49 & 3.48 \\
\hline \multirow[t]{2}{*}{ Chile } & Sin & 4.82 & 4.52 & 1.57 & 1.21 & 3.42 & 1.59 & 1.97 \\
\hline & Con apertura & 4.67 & 6.43 & 5.12 & 5.91 & 6.35 & 2.49 & 2.79 \\
\hline \multirow[t]{2}{*}{ México } & Sin & 2.75 & 8.70 & 1.57 & 3.77 & 6.59 & 1.59 & 2.83 \\
\hline & Con apertura & 2.56 & 5.82 & 5.12 & 4.96 & 5.75 & 2.49 & 2.38 \\
\hline \multirow[t]{2}{*}{ Perú } & $\operatorname{Sin}$ & 1.05 & 0.13 & 1.57 & 4.29 & 0.09 & 1.59 & 3.01 \\
\hline & Con apertura & 5.01 & 6.53 & 5.12 & 4.38 & 6.45 & 2.49 & 2.13 \\
\hline \multirow[t]{2}{*}{ Paraguay } & $\operatorname{Sin}$ & 3.62 & 1.45 & 1.57 & 1.13 & 1.10 & 1.59 & 1.94 \\
\hline & Con apertura & 2.90 & 5.72 & 5.12 & 6.06 & 5.65 & 2.49 & 2.85 \\
\hline \multirow[t]{2}{*}{ Uruguay } & Sin & 2.28 & 2.74 & 1.57 & 2.80 & 2.08 & 1.59 & 2.51 \\
\hline & Con apertura & 2.72 & 4.35 & 5.12 & 6.00 & 4.30 & 2.49 & 2.83 \\
\hline \multirow[t]{2}{*}{ Venezuela } & $\operatorname{Sin}$ & 1.28 & 4.31 & 1.57 & 0.52 & 3.26 & 1.59 & 1.74 \\
\hline & Con apertura & 2.75 & 1.69 & 5.12 & 7.16 & 1.67 & 2.49 & 3.33 \\
\hline Especificación & & $\log -\log$ & & & & ecimient & & \\
\hline Elasticidades & $\eta$ & $\psi$ & $\varepsilon$ & $\pi$ & $\eta$ & $\psi$ & $\varepsilon$ & $\pi$ \\
\hline $\operatorname{Sin}$ & 0.00 & -0.38 & 0.5 & 0.9 & 0.33 & 0.00 & 1.62 & 2.88 \\
\hline Con apertura & -0.35 & -0.48 & 2.86 & 1.36 & -0.25 & -0.46 & 2.94 & 2.87 \\
\hline
\end{tabular}

Nota: Elaborado con las elasticidades que se presentan en el cuadro 4, y las tasas de crecimiento económico nacional, de las exportaciones, importaciones, de la economía líder de la región, los precios internos, externos y los tipos de cambios correspondientes.

\section{Conclusiones}

Con los resultados de esta investigación se pudo comprobar que el crecimiento económico con restricciones por Balanza de Pagos se observó tanto en los países de la Unión Europea como de América Latina en los periodos previos y de integración económica y de apertura comercial en cada región respectivamente; 1957-1968 y 1969-1992 en la Unión Europea y 1980-1994 y 19952011 para América Latina. También se pudo constatar que la integración económica ayudó a aliviar los desequilibrios de balanza de pagos en los países europeos. De la misma manera que los resultados de Bairam (1988), con nuestra investigación se puede concluir que la experiencia Europea muestra diferentes resultados entre la tasa de crecimiento observada en comparación con la potencial que fue compatible con balanza de pagos y aunque la mayoría de los países presentaron tasas de crecimiento económico menor en el periodo de integración económica, resultaron no muy diferentes de la tasa compatible con equilibrio de balanza de pagos. Pocos países, como Luxemburgo y Holanda, crecieron a tasas de corto y largo plazo ligeramente por arriba de potencial compatible con 
el equilibrio de la balanza de pagos. Contrario a lo encontrado por Thirlwall y Pacheco (2006), en nuestros resultados se muestra una experiencia para los países de América Latina en la que todos, con excepción de Venezuela, logran mejorar su tasa compatible con el equilibrio de balanza de pagos. Nuestras estimaciones, muestran como la sensibilidad de las exportaciones al crecimiento de la economía estadounidense (elasticidad ingreso externo) aumenta, mientras que la propensión a importar permaneció prácticamente sin grandes cambios al comparar los periodos de apertura comercial con respecto al previo.

Los procesos de integración en la UE y AL fueron no solamente temporalmente distintos sino que también estuvieron sujetos a las características geográficas propias de cada región y de cada uno de los países participantes. Mientras que en la UE los flujos comerciales derivados de la integración económica estaban orientados principalmente a los sectores industrial (con la creación de la CEE), energético (con la creación de la Comunidad Europea de la Energía Atómica Euratom-) y la idea esencial sobre la libre movilidad de las personas. En AL se dirigieron al sector primario, pero en una etapa inicial de preferencia o liberalización arancelaria más que de libre comercio puro.

Los resultados de la investigación sugieren que la etapa de preferencias arancelarias en Europa (periodo 1957 1968) generó dependencia económica y fuertes relaciones comerciales de los miembros con relación al país líder (Alemania) y principal socio comercial, puesto que el desempeño de la economía alemana provocó un efecto de arrastre importante hacia el resto de los miembros (elasticidad ingreso de las exportaciones de 1.73). La liberalización comercial completa iniciada en 1968 estrechó los lazos comerciales ya existentes entre los miembros y funcionó como detonador del crecimiento de las exportaciones y del incremento del comercio entre los países de la CEE ligados con el desempeño económico del país líder (elasticidad ingreso de las exportaciones de 2.54).

La insipiente integración económica en AL entre 1980 y 1994 no permitió, de acuerdo con los resultados, desarrollar un verdadero comercio entre los miembros firmantes de los diversos acuerdos comerciales. La poca planeación y la complicada toma de decisiones en cuanto a los productos que se podrían desgravar así como el poco volumen de comercio entre los miembros de la ALADI y la inexistencia de in país líder provocaron el desinterés del bloque económico por parte de los miembros debilitando el comercio, por esta razón la elasticidad ingreso de las exportaciones es de sólo 0.55 , inelástica. A partir de 1994 la aparición de otros bloques o tratados comerciales como el G-3, el Mercosur y el TLCAN, a pesar de tratarse en su mayoría de zonas de preferencias arancelarias, tomó en consideración las características geográficas y las ventajas de cada país generando sinergias y compromisos importantes que llevaron a la creación de lazos comerciales importantes entre ellos elevando la elasticidad ingreso de las exportaciones hasta 2.86 convirtiendo con esto a las exportaciones en el motor del crecimiento regional.

La integración económica europea entre 1957 y 1968 hizo posible establecer y consolidar vínculos comerciales importantes entre los miembros de la CEE, a partir de 1969 esos lazos se estrecharon, fortalecieron,+ consolidaron y estabilizaron con el libre comercio. Mientras que en AL no existió, en la práctica, integración económica ni comercio hasta 1994 en que se establecen diversas áreas de preferencias arancelarias planeadas pero que hasta ahora no 
se han consolidado.

La integración económica y su consiguiente apertura comercial beneficia a los miembros al permitirles el acceso a mercados más grandes y expandir con ello su aparato exportador incrementando su crecimiento potencial compatible con la balanza de pagos. Sin embargo, aunque los procesos en ambas zonas fueron diferentes, cada uno tiene sus características particulares. Ambos procesos permitieron aliviar en buena medida las restricciones por balanza de pagos. El proceso de la UE ofreció la ventaja de la buena planeación, maduración y consolidación con tasas de crecimiento moderadas (sin llegar al potencial) mientras que el desordenado proceso inicial en AL les permitió a los integrantes un mayor crecimiento (sin llegar a su potencial) pero sin llegar a su etapa de consolidación. Las medidas más recientes aplicadas por la UE sobre el desarrollo de infraestructura, atracción de inversión y creación de empleos podrían hacer de este modelo el más exitoso, a pesar de ello, no se puede aseverar categóricamente que sea mejor que la integración en AL.

De la misma manera que la mayoría de los estudios empíricos, encontramos que los precios relativos no influyen de manera relevante tanto en las exportaciones como en las importaciones en los periodos de integración y apertura comercial en los países tanto de la Unión Europea como de América Latina. Por lo anterior resultó más adecuado analizar bajo el enfoque del multiplicador dinámico del comercio de Harrod en sus dos versiones; relación de tasa de crecimiento de las exportaciones con respecto a la elasticidad ingreso de las importaciones y la versión con elasticidad ingreso externo, crecimiento del ingreso de la economía líder con respecto a la elasticidad ingreso de las importaciones. Esto es muy importante, debido a que establece que el comportamiento del flujo de exportaciones e importaciones, no depende de las políticas enfocadas en modificar los precios. Y, aunque los países de América Latina han logrado aliviar en buena medida los desequilibrios de balanza de pagos desde que forman parte de alguna integración económica, la madurez y consolidación de la misma que permita el crecimiento de las exportaciones podría lograr aumentar el crecimiento potencial, siempre tomando en cuenta el desempeño de la economía del país líder. Políticas tendientes reducir las desigualdades económicas y sociales entre los países miembro así como la libre movilidad de los factores podrían funcionar como lubricante para la producción y comercio y el funcionamiento más eficiente al interior de la región.

\section{Bibliografía}

Alonso, J., \& Garcimartín, C. (1999). A New Approach to Balance of Payments Constraint: Some Empirical Evidence. Journal of Post Keynesian Economics, pp. 259-282.

Ashok, P. (2006). Relationship between Trade Liberalization, Growth, and Balance of Payments in Developing Countries: An Econometric Study. The International Trade Journal, XX(4), pp. 429-467.

Atesoglu, H. (Junio de 1994). Balance of Payments Determinated Growth in Germany. Applied Economic Letter, I, pp. 89 - 91.

Bairam, E. (1988). Balance of Payments, the Harrod Foreign Trade Multiplier and Economic Growth: The European an North American experience, 1970 - 1985. Applied Economics, pp. 1635-1642.

Bairam, E. (1993). Static versus Dynamic Specification and the Harrod Foreign Trade Multiplier. Applied Economics, (25), pp. 739-742. 
Bajo-Rubio, O., \& Díaz, C. (2009). Does the Balance of Payments Constrain Economic Growth? Some Evidence for the New EU Members. Post Communist Economies, XXI(1), pp. 41-46.

Calvo, A. (2003). Integración económica y regionalismo. Principales acuerdos regionales. Madrid: Centro de estudios Ramón Areces, S. A.

Chang, Y. (2004). Bootstrap Unit Root Tests in Panels with Cross-Sectional Dependency. Journal of Econometrics, 120, pp. 263-293.

Feenstra, R. C., Inklaar, R., \& Timmer, M. (2015). The Next Generation of the Penn World Table. Forthcoming American Economic Review.

Ferreira, A. \& Canuto, O. (2003). Thirlwalls Law and Foreing Capital in Brazil. Momento Económico, 125, pp. 18-29.

Funck, B., \& Pizzati, L. (2003). European Integration, Regional Policy and Growth. Washington: World Bank.

Gairuzazmi, M. (2006). Balance of Payments Constrained Growth Model: An Examination of Thirlwalls Hypothesis using McCombies Individual Country Method. Applied Economics Letters, (13), pp.763-768.

Gazol, A. (2008). Bloques Económicos. México: Facultad de Economía, UNAM.

Houthakker, H., \& Magee, S. (1969). Income and Price Elasticities in World Trade. Review of Economics and Statistics.

Im, K., Pesaran, M., \& Shin, Y. 2003. Testing for Unit Roots in Heterogeneous Panels. Journal of Econometrics, pp. 53-74.

Landesmann, M., \& Pöschl, J. (1996). Balance of Payments Constrained Growth in Central and Eastern Europe and Scenarios of East - West Integration. Russian and East European Finance and Trade, pp. 30-84.

Lanzafame, M. (2014). The Balance of Payments Constrained Growth Rate and the Natural Rate of Growth: New Empirical Evidence. Cambridge Journal of Economics, 38, pp. 817-838.

Levin, A., Lin, C., \& Chu, C. (2002) Unit Root Tests in Panel Data: Asymptotic and Finite Sample Properties, Journal of Econometrics, 124.

McCombie, J. S. (1997). On the Empirics of Balance of Payments Constrained Growth. Journal of Post Keynesian Economics, 19(3), pp. 345-375.

McCombie, J. S. (1997). The Dynamic Harrod Foreign Trade Multiplier and the Demand Oriented Approach to Economic Growth: An Evaluation. International Review of Applied Economics, (11), 5 - 26.

Moreno Brid, J. C., \& Pérez, E. (1999). Balance of Payments Constrained Growth in Central America: 1950 - 1996. Journal of Post Keynesian Economics, (1), pp. 131-147.

Morones, A. (2016). Crecimiento económico en México: restricción por la Balanza de Pagos. Ensayos Revista de Economía, XXXV(1), pp. 39-58.

Muñiz, J. (2005). Lecturas de integración económica. La Unión Europea. España: Edicions Universitat de Barcelona.

Ocegueda, J. M. (2007). Apertura comercial y crecimiento económico en las regiones de México. Investigación Económica, LXVI(262), pp. 89-137.

Pastor, R. (1993). Integration with Mexico. New York: The Twentieth Century Fund Press.

Persyn, D. \& Westerlund, J. (2008). Error Correction Based Cointegration Tests for Panel Data. The STATA Journal, 8(2), pp. 232-241.

Santos, P., \& Thirlwall, A. P. (1999). The Impact of Trade Liberalization on Export Growth, Import Growth the Balance of Trade and the Balance of Payments of Developing Countries. Kent: Mimeo.

Schiff, M., \& Winters, A. (2003). Integración regional y desarrollo. Colombia: Alfaomega.

Tamames, R. (2006). Estructura económica internacional. Madrid: Alianza Editorial.

Taylor, M. P. (1993). Modeling the Demand of U. K. Money, 1871 - 1913. Review of Economics ans Statistics, pp. 112-117.

Thirlwall, A. P. (1979). The Balance of Payments Constrains as an Explanation of International Growth Rate Differences. Banca Nazionale del Lavoro Quarterly Review, (128), pp. 45-53. 
52 Nueva Época REMEF (The Mexican Journal of Economics and Finance)

Thirlwall, A. P. (1997). Reflections on the Concept of Balance of Payments Constrained Growth. Journal of Post Keynesian Economics, 19(3), pp. 377-385.

Thirlwall, A. P. (2003). Growth and Development. With Special Reference to Developing Economies. New York: Palgrave.

Thirlwall, A. P., \& Pacheco, P. (2006). Trade Liberalization, the Income Elasticity of Demand for Imports, and Growth in Latin America. Journal of Post Keynesian Economics, 29(1), pp. $41-66$.

Westerlund, J. (2007). Testing for Error Correction in Panel Data. Oxford Bulletin of Economics ans Statistics, 69, $709-748$. 